We assessed cannulation success, time to cannulate, and correct treatment identification for each participant.

Results 14 experienced (9 Fellows, 5 Consultants) and 9 junior (Registrars) medical staff attempted 46 cannulations. Experienced participants successfully cannulated $100 \%$ of treated and control sections with no significant difference in mean (SD) time to cannulate (98 (75)s and 97(51)s respectively, $p=0.97)$. Junior participants cannulated $89 \%$ and $67 \%$ of treated and control sections respectively $(\mathrm{p}=0.69)$, and mean $(\mathrm{SD})$ time to cannulate was 132 (78)s and 106 (53)s respectively ( $p=0.42)$. GTN treated arteries were correctly identified by $43 \%$ of experienced and $22 \%$ of junior participants $(\mathrm{p}=0.47)$.

Conclusions This study suggests that topical application of GTN does not increase successful cannulation of umbilical arteries by experienced staff. More participants or prolonged GTN application time may be needed to confirm these findings in junior staff.

\section{PS-199 IMPROVED IRRADIANCE OF PHOTOTHERAPY DEVICES IN DUTCH NEONATAL INTENSIVE CARE UNITS}

SJ Van 't Klooster, KE Lorenz, CV Hulzebos, PH Dijk. Pediatrics, Beatrix Children's Hospital/ University Medical Center Groningen, Groningen, Netherlands

\subsection{6/archdischild-2014-307384.495}

Background/aims Phototherapy (PT) is an effective treatment for hyperbilirubinemia, provided a minimum irradiance level is applied. Previously, we reported on low irradiance levels of PT devices in Dutch Neonatal Intensive Care Units (NICUs). These data were shared with all NICUs. We hypothesised that this knowledge would positively affect current applied irradiance levels. Therefore we determined irradiance levels of PT devices again in 2013.

Methods Irradiance levels of overhead and underneath PT devices in all 10 NICUs were measured with a Dale 40 radiometer (FlukeBiomedical, Everett, Washington, USA), in routinely applied PT practice patterns, using an infant silhouette model. The infant's distance from the overhead device was measured.

Results Irradiance levels of 35 PT device-incubator combinations were measured (Table); 10 types of PT devices were in use in the 10 NICUs (8 overhead and 2 underneath). Overall irradiance levels increased ( $p=0.01)$; irradiance levels of overhead and underneath PT devices also increased with 50\% (NS) and 200\% $(\mathrm{p}=0.03)$, respectively. The mean (range) distance between overhead PT device and infant decreased with $7 \mathrm{~cm}$ to 38 (3062) $\mathrm{cm}(\mathrm{p}<0.01)$. Minimal recommended irradiance levels of $10 \mu \mathrm{W} / \mathrm{m}^{2} / \mathrm{nm}$ were obtained for $70 \%$ of PT devices versus $\sim 50 \%$ in 2008 ( $\mathrm{p}=0.02$ ).

Conclusions Applied irradiance levels of PT devices in Dutch NICUs have markedly improved in 2013. Current data suggest that awareness among healthcare workers regarding requirements for effective PT results in improved use of PT devices, including smaller distances between PT device and infant.

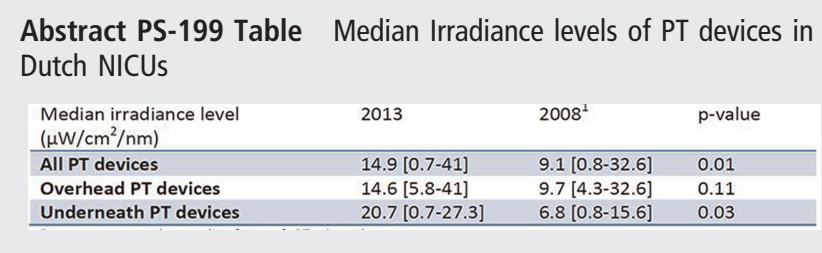

Moreover, the availability of better performing (Light Emitting Diode) PT devices might have contributed.

\section{PS-200 HOSPITAL-LEVEL VARIATION IN RADMISSION RATES OF NEONATAL INTENSIVE CARE (NICU) PATIENTS: A POTENTIAL QUALITY MEASURE}

S Lorch, M Macheras. Pediatrics, The Children's Hospital of Philadelphia, Philadelphia, USA

\subsection{6/archdischild-2014-307384.496}

Background Variation in readmission rates may assess the quality of a provider through the quality of inpatient care or transitions from inpatient to outpatient providers. The aim of this project was to validate readmission rates as a measure of NICU quality.

Methods Using birth certificates linked to maternal and infant hospital discharge records, a cohort was constructed of 23-34 week gestation infants who survived to hospital discharge at a California hospital discharging over 50 such infants per year between 1995-2009 ( $\mathrm{N}=296,509$ at 141 hospitals). Unadjusted variations in hospital readmission rates within 7, 14, 30, 90, and 365 days after discharge were compared to rates adjusted for hospital casemix, including patient gestational age, insurance status, race/ethnicity, and maternal education, and BPD, IVH, NEC, and ROP as measures of chronic health conditions.

Results Unadjusted readmission rates varied significantly between hospitals and across geographic regions, ranging from 2.2-28.4\% 7-14 days after discharge to $2.7-34.4 \% 365$ days after discharge. Some of this variation was explained by hospital casemix. However, after risk adjustment, there remained a 7.911.5 fold difference in readmission rates between hospitals with the lowest and highest rates across the five time frames that did not change when complications of preterm birth were included in the risk-adjustment model.

Conclusions There is substantial variation in readmission rates of premature infants that is only partially explained by gestational age and social factors. Readmission rates may provide a measure of the quality of NICU care and the integration of services within a geographic area.

\section{PS-201 ASSOCIATION BETWEEN BLOOD TRANSFUSION AND DEVELOPMENT OF RETINOPATHY OF PREMATURITY - SYSTEMATIC REVIEW OF LITERATURE AND META-ANALYSIS}

1J Banerjee, ${ }^{2} \mathrm{~F}$ Asamoah, ${ }^{2} \mathrm{JK}$ Morris, ${ }^{3} \mathrm{~N}$ Aladangady. ${ }^{1}$ Neonatology, Homerton University Hospital, London, UK: ${ }^{2}$ Department of Medical Statistics, Wolfson Institute for Preventive Medicine Barts and the London School of Medicine and Dentistry QMUL, London, UK; ${ }^{3}$ Department of Neonatology, Homerton University Hospital, London, UK

\subsection{6/archdischild-2014-307384.497}

Background and aims Retinopathy of Prematurity (ROP) is an important cause of visual impairment and blindness in children. ${ }^{1}$ Aim Conduct a systematic review and meta-analysis to determine the association between blood transfusion and the development of ROP in preterm infants $\leq 32$ weeks gestational age or birthweight $<1500$ grams.

Methods

Data Sources: MEDLINE, EMBASE, Cochrane Register, CINAHL, LILACS, Web of Knowledge, clinicaltrial.gov and Open SIGLE. 